\title{
Recent Achievements on the Development of the HERSCHEL/PACS Bolometer arrays
}

\author{
Billot N. ${ }^{\mathrm{a}, *}$ Agnèse P. ${ }^{\mathrm{b}}$ Boulade O. ${ }^{\mathrm{a}}$ Cigna C. ${ }^{\mathrm{b}}$ \\ Doumayrou E. ${ }^{a}$ Horeau B. ${ }^{\text {a }}$ Lepennec J. ${ }^{\text {a }}$ Martignac J. ${ }^{a}$ \\ Pornin J.-L. ${ }^{b}$ Reveret V. ${ }^{\text {a }}$ Rodriguez L. ${ }^{a}$ Sauvage M. ${ }^{\text {a }}$ \\ Simoens F. ${ }^{b}$ Vigroux L. ${ }^{a, c}$ \\ ${ }^{\text {a } C E A / S a c l a y / S A p, ~ U M R ~ C E A / C N R S / U P 7 ~ L a b o r a t o i r e ~ A I M, ~ B a t . ~ 709, ~ l ' O r m e ~}$ \\ des merisiers, 91191 Gif-sur-Yvette, France \\ ${ }^{\mathrm{b}}$ CEA/LETI Grenoble, 17 Avenue des Martyrs, 38054 Grenoble, France \\ 'Institut d'Astrophysique de Paris, 75014 Paris, France
}

\begin{abstract}
A new type of bolometer arrays sensitive in the far Infrared and Submillimeter range has been developed and manufactured by CEA/LETI/SLIR since 1997. These arrays will be integrated in the PACS instrument (Photodetector Array Camera and Spectrometer) of ESA's Herschel Space Observatory (launch date 2007). The main innovations of CEA bolometers are their collective manufacturing technique (production of 3-side buttable 16x16 arrays) and their high mapping efficiency (large format detector and instantaneous Nyquist sampling). The measured NEP is $2.10^{-16} \mathrm{~W} / \sqrt{\mathrm{Hz}}$ and the thermometric passband about $4-5 \mathrm{~Hz}$. In this article we describe CEA bolometers and present the results obtained during the last test campaign.
\end{abstract}

Key words: Submillimeter astronomy, Filled bolometer arrays, All-Si design, Multiplexed readout, monolithic array, NEP

\section{Introduction}

The Herschel Mission stands as the next great step in FIR and submillimeter astronomy. This huge satellite will hold three scientific instruments (HIFI, SPIRE and PACS (1)) and the largest telescope ever sent in space $(3.5 \mathrm{~m}$ in

\footnotetext{
* Corresponding author: Tel.: +33 (0)169 089 570; Fax: +33 (0)169 086577
}

Email address: nbillot@cea.fr (Billot N.). 
diameter). It will explore a universe that is unobservable from the ground. The space observatory will carry out large scale surveys of the distant Universe to study galaxy evolution. It will also focus on our own Galaxy to inform us on the composition, chemistry and life-cycle of the interstellar medium. Targets like comets, solar system planets and extra-solar planetary disks are also foreseen. The Herschel satellite is due to launch in 2007 to reach the second Lagrangian point L2 after a 6 months journey. The mission is funded by the European Space Agency and has a nominal duration of 3.5 years. CEA/DAPNIA and CEA/LETI have developed a very sensitive camera made up of more than 2500 micro-bolometers (operating at $300 \mathrm{mK}$ ) for use in the PACS photometer (60-210 microns).

During summer 2005, we have run performance measurements at CEA Saclay to qualify the Bolometer Focal Plane (BFP) before delivering it to MaxPlanck-Institut fur extraterrestrische Physik (MPE) Garching by the end of the year for integration in the PACS instrument.

Section 2 is an outline of the detector design while section 3 concentrates on the latest results obtained during the qualifying test campaign.

\section{PACS bolometers}

PACS photometer uses a dichroic to split light between two channels dedicated to short- $(60-130 \mu m)$ and long-wavelengths $(130-210 \mu m)$. The two focal planes have the same field of view $(3.5 \times 1.75 \mathrm{arcmin})$ and consist of tiled matrices manufactured by blocks of $16 \times 16$ pixels and for the first time in bolometers' history, the cold readout electronics circuit (300 mK) includes a 16 to 1 multiplexing function. The detector is based on an all-Silicon technology development that takes advantage of Si micro-machining techniques maturity. Unlike most current submillimetric imagers, CEA bolometers do not use Winston cones as light concentrators but rather a reflecting plate and a quarterwave cavity to optimise absorption. All these features made it possible to build detectors with a large number of small contiguous pixels. Actually twelve of these pixels fit in the Airy disc of the telescope (pixel width $=750 \mu m=$ $0.5 F \lambda$ ); which is, according to sampling theory, enough to correctly sample the Point Spread Function (PSF) in a single shot (no need for jiggling observing mode). This makes the camera very efficient at mapping large areas of the sky.

The simplest way to describe the PACS bolometers is to divide each pixel in a detection layer which comprises the suspended absorbing grid, the thermometric sensor and the Si interpixel wall on the one hand; and a readout layer that contains the CMOS multiplexer and the reflecting gold sheet on the other hand. The two layers are hybridised with $20 \mu \mathrm{m}$ Indium bumps that ensure electrical and thermal contacts (see figure 1). The bolometer signal is read at 


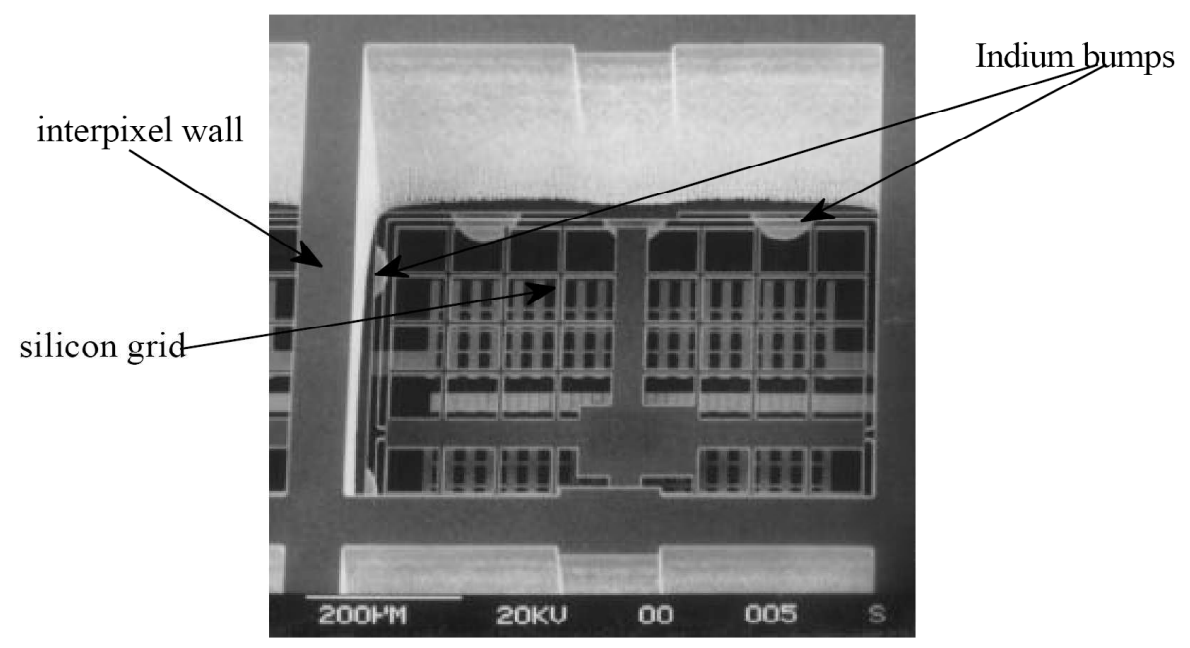

Fig. 1. Microphotography of a single pixel $(750 \times 750 \mu m)$.

the middle point of a resistor bridge; one resistor is located in the interpixel wall and held at bath temperature $(\sim 300 \mathrm{mK})$ and the other is implanted in a mesa configuration in the middle of the absorber.

Thermometers are very high impedance resistors (about $4 \mathrm{G} \Omega$ ) working in the 'hopping conduction' regime at $300 \mathrm{mK}$. Such a high impedance is achieved by ion implantation ( $100 \%$ phosphorus compensated with $50 \%$ boron) on a double Silicon-On-Insulator (SOI) substrate. They exhibit an exponential dependence on temperature. Non-ohmic effects are minimised for long linear thermometer geometry.

EM absorption takes places at the top of a quarter-wave resonant cavity and reaches nearly $100 \%$ efficiency when the absorber (TiN deposited on Si grid) is put where the antinode of the stationary wave is created. The size of the cavity is tuned with the Indium bumps diameter (20 $\mu \mathrm{m}$ in this case).

The cold electronics is located just below the $\lambda / 4$ cavity reflector to minimise stray capacitances from electronics lines. A CMOS multiplexer (inspired by ISOCAM technology (2)) is also implemented to reduce dissipation at the $300 \mathrm{mK}$ stage and increase the potential number of pixels in the confined focal plane. It offers a 16 to 1 MUX function.

More details on the detector design or on mechanical and thermal issues may be found in (יㅡ).

Herschel's telescope will be passively cooled to $80 \mathrm{~K}$ and should have a relatively low emissivity (about 4\%). The blackbody emission from the dish will represent most of the flux illuminating the detector and we expect astronomical sources to be a percent or less of the background flux. The requirement on sensitivity (photon noise limited) is $5 \mathrm{mJy}(5 \sigma, 1$ hour) which is equivalent to a Noise Equivalent Power (NEP) of $\sim 1.5 \times 10^{-16} \mathrm{~W} / \sqrt{\mathrm{Hz}}$, depending on efficiency assumptions. 


\section{Performance tests}

The experimental setup in Saclay consists in a cryostat, an ${ }^{3} \mathrm{He}$ sorption cooler and two regulated blackbodies. The illumination of the detector is usually modulated between the two blackbodies to mimic chopped observations. The background flux is set to $2 \mathrm{pW} /$ pixel corresponding to a telescope at $80 \mathrm{~K}$.

During the last test campaign we quantified the noise level, the responsivity and the thermometric passband. We carried out systematic measurements of these parameters as a function of the bias voltage applied across the resistor bridge for two different background fluxes. All measurements were obtained in the nominal readout mode (differential readout). The signal was sampled at the nominal readout frequency of $40 \mathrm{~Hz}$.

The noise spectral density exhibits two distinct components: a low-frequency noise spectrum due to electronics and temperature drifts and a white noise spectrum at higher frequencies. The $1 / f$ knee frequency occurs at about $0.1-1 \mathrm{~Hz}$ and the noise level measured at $3 \mathrm{~Hz}$ is about $9 \mu \mathrm{V} / \sqrt{\mathrm{Hz}}$.

The responsivity is measured to be about $4 \times 10^{10} \mathrm{~V} / \mathrm{W}$ with a background flux of $2 \mathrm{pW} /$ pixel and a modulation of $0.5 \mathrm{pW}$ (figure 2). Such high responsivities are required to overcome the relatively high noise level due to the cold CMOS held at $300 \mathrm{mK}$.

The NEP is actually a figure of sensitivity and is defined as $\frac{\text { noise }}{\text { responsivity }}$. We find an optimal NEP of $2.2 \times 10^{-16} \mathrm{~W} / \sqrt{\mathrm{Hz}}$ for a voltage bias of $2.8 \mathrm{~V}$.

The thermometric passband is defined as being the modulating frequency at which the signal is attenuated by $3 \mathrm{~dB}$. It is a crucial parameter that will constrain the maximum scanning speed of the telescope. The measurements gave a passband of $4-5 \mathrm{~Hz}$.

Since Herschel will be under the influence of ionising particles from the Solar wind, the detector was also tested for irradiation. It received a cumulated dose of $20 \mathrm{krad}$ of $\gamma$-rays ( 10 years in space) and different fluxes of protons $(20 \mathrm{MeV})$ and alpha particles $(30 \mathrm{MeV})$ without degradation to the performances (upset time $<200 \mathrm{~ms}$ ).

\section{Prospects}

The next step is the PACS calibration campaign that will take place at MPE Garching for 6 months before delivery to ESA in mid-2006. Meanwhile, CEA bolometers are being optimised for longer wavelengths to match atmospheric windows. This way, they could be used on ground telescopes like KOSMA, APEX or IRAIT at Concordia Station in Antarctica (4). 


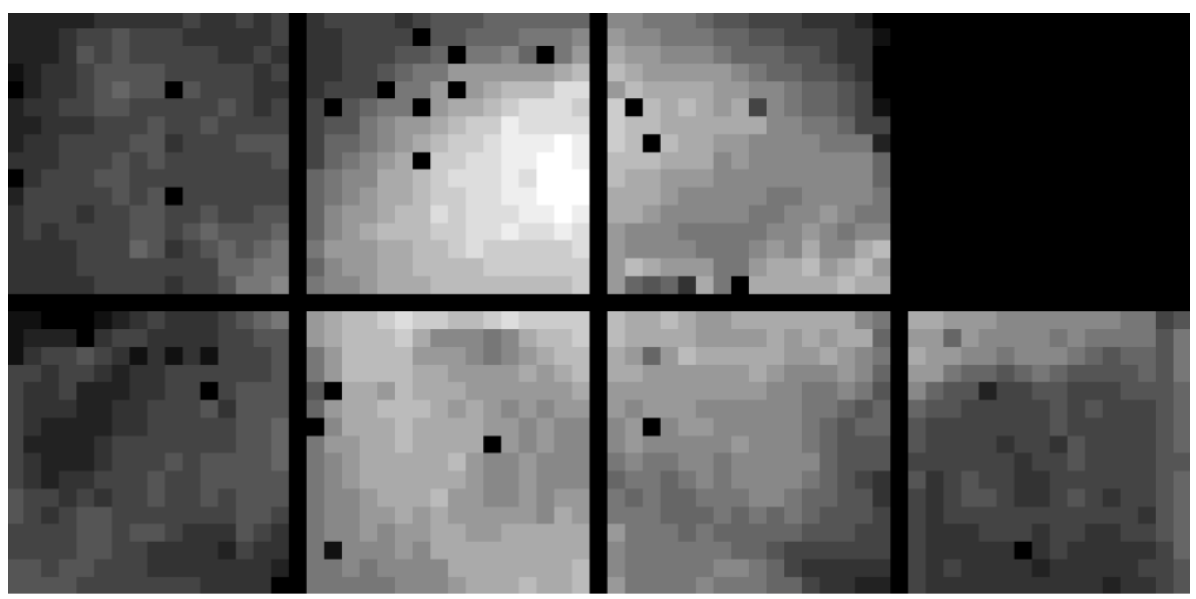

Fig. 2. Responsivity map of the short-wavelength BFP (2pW/pixel background and $500 \mathrm{fW}$ modulation). The circular central feature in the map is due to internal reflexions inside the cryostat. Finer measurements will be done at MPE using the two Internal Calibration Sources inside PACS. Dead pixels represent less than $2 \%$ of the total number of pixels. The missing array was not functional at the time of the test but has been repaired and is currently being tested.

\section{References}

[1] Pilbratt, Goran. Herschel SPACE Observatory mission summary, Exploiting the ISO Data Archive. Infrared Astronomy in the Internet Age, held in Siguenza, Spain 24-27 June, 2002. Published as ESA Publications Series, ESA SP-511. European Space Agency,2003, p.31.

[2] Cesarsky C. et al Development of ISOCAM : The camera of the Infrared Space Observatory, Infrared Spaceborne Remote Sensing. Proceedings of the SPIE, Volume 2019, pp. 36-47 (1993).

[3] Agnèse P. et al Filled bolometer arrays for Herschel/PACS, Millimeter and Submillimeter Detectors for Astronomy. Proceedings of the SPIE, Volume 4855, pp. 108-114 (2003).

[4] Busso M. et al The IRAIT Project Infrared Astronomy from Antarctica, European Astronomical Society Publications Series, Volume 14, 2005, pp.181-186. 